\title{
Ultrasound-guided injections of hyperosmolar dextrose for overuse patellar tendinopathy: a pilot study
}

\author{
Michael Ryan, ${ }^{1}$ Anthony Wong, ${ }^{2}$ David Rabago, ${ }^{3}$ Kenneth Lee, ${ }^{4}$ Jack Taunton ${ }^{5}$
}

'Department of Orthopedics and Rehabilitation, University of Wisconsin, Madison, Wisconsin, USA

2Department of Radiology, St Paul's Hospital, Vancouver, Canada

${ }^{3}$ Department of Family Medicine, University of Wisconsin, Madison, Wisconsin, USA

${ }^{4}$ Department of Radiology, University of Wisconsin, Madison, Wisconsin, USA ${ }^{5}$ Division of Sports Medicine, University of British Columbia, Vancouver, Canada

\section{Correspondence to}

Dr Michael Ryan, 4195 Medical

Sciences Center, 1300

University Avenue, Madison, WI 53706, USA;

mryan76@gmail.com

Accepted 19 January 2011 Published Online First 21 February 2011

\begin{abstract}
Purpose To evaluate whether ultrasound-guided injection of hyperosmolar dextrose for treatment of patellar tendinopathy decreases pain scores and normalises the appearance of the patellar tendon on ultrasound.

Methods Subjects were referred from primary care clinics and failed conservative treatment. Subjects received a diagnostic ultrasound examination, then ultrasound-guided injection of $25 \%$ dextrose with lidocaine into the area of tendinopathy until they were satisfied with treatment. The primary outcome measure was a three-part visual analogue scale (VAS; baseline and mean of 45 weeks after start of treatment) for pain at rest, activities of daily living (ADL) and during sport. Secondary outcomes included segmental ultrasound examinations assessing tendon hypoechogenicity (area and severity score), neovascularity (severity score) and the presence or absence of intratendinous tearing and calcification, irregularities of cortical bone and thickness. Results 47 consecutive referrals were included. Subjects received a mean of four $( \pm 3)$ injection sessions. At mean 45 weeks post-enrollment, subjects reported a reduction in pain across the three VAS items (rest 38.4 $\pm 25-18.7 \pm 18.4$; ADL 51.1 $\pm 22.9-25.8 \pm 20.1$; sport 78.1 $\pm 15.7-38.8 \pm 26.1 ; p<0.01)$. There was improvement in neovascularity following the dextrose injection. A significant correlation between hypoechogenicity severity scores and pain at follow-up is reported.

Conclusion There was a reduction in pain and an improvement in ultrasound appearance following ultrasound-guided dextrose injections for refractory patellar tendinopathy. An improved hypoechoic appearance of the tendon was associated with decreased pain scores, suggesting that dextrose injections may modify patellar tendinopathy at the tissue level and that fibrillar changes may play a role in tendon nociception.
\end{abstract}

Patellar tendinopathy ('jumper's knee') is a painful and disabling condition caused by chronic repetitive stress of the proximal patellar tendon at its insertion on the inferior pole of the patella. ${ }^{1}$ It is particularly prevalent in athletic populations, affecting $40-50 \%$ of persons in those sports requiring repetitive, explosive power from the knee extensors, including basketball, volleyball, soccer and track and field. ${ }^{2}$ Patellar tendinopathy is associated with significant impact on quality of life, loss of function and changes on ultrasound imaging of the tendon. ${ }^{3}$ Whereas current therapy is associated with the resolution of patellar tendinopathy for many, patellar tendinopathy can become chronic in a subset of patients, severely

\section{What is already known on this topic}

- Chronic infrapatellar tendinopathy presents a challenge to the physician, especially after failing conservative exercise-based treatments.

- No single therapy has been identified.

- Dextrose injection treatments show promise for helping reduce pain at the plantar fascia, Achilles and adductor tendon injury sites.

\section{What this study adds}

- After ultrasound-guided dextrose injections the pain associated with infrapatellar tendinopathy and the sonographic appearance was improved.

- Tendinopathic pain was associated with the ultrasound echo pattern during the treatment course.

impacting an individual's long-term participation in sport. Half of all cases of patellar tendinopathy managed conservatively become chronic and are prone to recur; an estimated $29 \%$ of patients become candidates for surgery upon failing conservative treatment. ${ }^{45}$ One prospective case-control analysis of athletes with patellar tendinopathy reported that more than $50 \%$ of patients with patellar tendinopathy ended their sports career as a result of this injury. ${ }^{6}$ No widely used therapy for patellar tendinopathy addresses the underlying tissue degeneration now understood to be the hallmark of overuse tendinopathies. ${ }^{7}$

Prolotherapy is an injection therapy used to treat chronic musculoskeletal pain including tendinopathies. ${ }^{8}$ It has been hypothesised to stimulate healing of chronic soft tissue injury by a local inflammatory response to therapy, although the precise mechanism is unclear. ${ }^{9}$ Prolotherapy using hypertonic dextrose has been reported to reduce pain associated with chronic tendinopathies at the Achilles tendon, plantar fascia and groin. ${ }^{10-12}$

Dextrose prolotherapy has also been associated with ultrasound-based changes associated with tissue healing in overuse tendinopathy. ${ }^{13}$ The histological, sonographic and clinical presentation of tendinopathy appears similar in 
cases at the Achilles, common elbow extensor and patellar tendons, ${ }^{2} 1415$ as well as the plantar fascia, ${ }^{12}$ and they are thought to share underlying degenerative pathophysiology. ${ }^{16}$ Although dextrose prolotherapy has shown clinical efficacy in reducing tendinopathic pain at other locations, it has not yet been assessed as a treatment for patellar tendinopathy. ${ }^{10} 1213$ Therefore, given the lack of a definitive treatment option for infrapatellar tendinopathy that is non-responsive to conservative measures, we conducted a prospective case series to address the hypotheses that ultrasound-guided dextrose injection therapy for patellar tendinopathy would be associated with a reduction in pain and ultrasound changes in tendon architecture consistent with tendon healing. A secondary hypothesis is that pain and sonographic features of tendinopathy are positively correlated following dextrose injection.

\section{MATERIALS AND METHODS \\ Participants}

The diagnosis of patellar tendinopathy was made clinically on the basis of self-reported pain and palpatory tenderness along the length of the patellar tendon at its proximal insertion directly at the inferior pole of the patella, and radiologically based on the presence of one or more of the following abnormalities: hypoechoic regions; discrete anechoic areas or intratendinous tears and neovascularisation. Participants in the study were referred from August 2003 to February 2009 by board-certified sports medicine specialists in the local Vancouver area after failing standard-of-care therapy including: eccentric drop-squats $(n=22)$; supervised general physiotherapy $(n=16)$; non-steroidal anti-inflammatory medication $(n=9)$; general exercises for knee extension and for the lower extremity $(n=8)$; extracorporeal shock wave therapy $(n=4)$; foot orthoses $(n=4)$; knee braces $(n=4)$; acupuncture $(n=2)$; chiropractic manipulation $(n=1)$ and cortisone injections $(n=1)$. The athletic backgrounds of the study population were varied: five performed their sport at a national level of competition $(n=1$, skiing; $\mathrm{n}=2$, running/track; $\mathrm{n}=1$, spring board diving; $\mathrm{n}=1$, ballet dance), seven were non-athletes (non-athletic activities not documented) and the remainder were active on a recreational basis.

The study protocols received ethical approval from the University of British Columbia Clinical Review Board.

\section{Ultrasound examination}

The ultrasound examination and the injection procedure were performed by the same radiologist (AW) with 20 years experience in musculoskeletal radiology and research. The patellar tendon and surrounding tissue was examined with the patient lying supine and the knee in a straight position. The ultrasound examination was performed using a Philips HDI 5000 ultrasound machine (Philips, Phillips USA, Bothell, Washington, USA) using both a 5-12 MHz and 7-15 $\mathrm{MHz}$ linear array high resolution transducer/receiver. The tendon in its entirety was examined in the longitudinal and transverse planes, with tendon thickness recorded as the greatest distance spanning the tendon in the anterior-posterior plane. Care was taken to image the patellar tendon parallel with the fibres in the longitudinal plane and perpendicular to the fibres in the transverse plane to avoid anisotropic artifacts. ${ }^{17} 18$ Colour flow Doppler (6.6 $\mathrm{MHz}$ ) was used to diagnose the extent of neovascularity on a modified Öhberg $0-3$ ordinal scale, on which ' 0 ' represented no blood vessels visible, ' 1 ' indicated mild (one to two vessels extending into the tendon), ' 2 ' moderate (three to four neovessels) and ' 3 ' marked (more than four vessels extending into the tendon) neovascularity (figure 1). ${ }^{19}$

Hypoechoic regions were documented in two ways. The first measured the cross-sectional area of the hypoechoic region in square millimetres using the free-hand measurement tool in Inteleviewer radiological software (version 3.5.1.P98, 2002; figure 2). ${ }^{20}$ The second evaluation of hypoechoic areas (or echotexture rating) used a novel 0-3 ordinal scale to document the severity of any given hypoechoic area, in which ' 0 ' represented normal echogenicity and fibrillar pattern, ' 1 ' represented predominantly signs of irregular collagen fibre pattern coupled with a slightly weakened echo signal, '2' represented significant hypoechoic regions and fibre irregularity, and ' 3 ' represented marked hypoechogenicity with anechoic regions. The length of all observable tears was measured in the longitudinal plane in millimetres (figure 3).

The presence or absence of intratendinous calcification was documented, as was irregularity of the cortical bone of the patella.

\section{Hyperosmolar dextrose injection}

Injections with $25 \%$ dextrose ( $1 \mathrm{ml} \%$ lidocaine $(20 \mathrm{mg} / \mathrm{ml})$ and $1 \mathrm{ml} 50 \%$ dextrose $(25 \mathrm{~g} / 50 \mathrm{ml}$ ) (dextrose monohydrate $500 \mathrm{mg}$ )

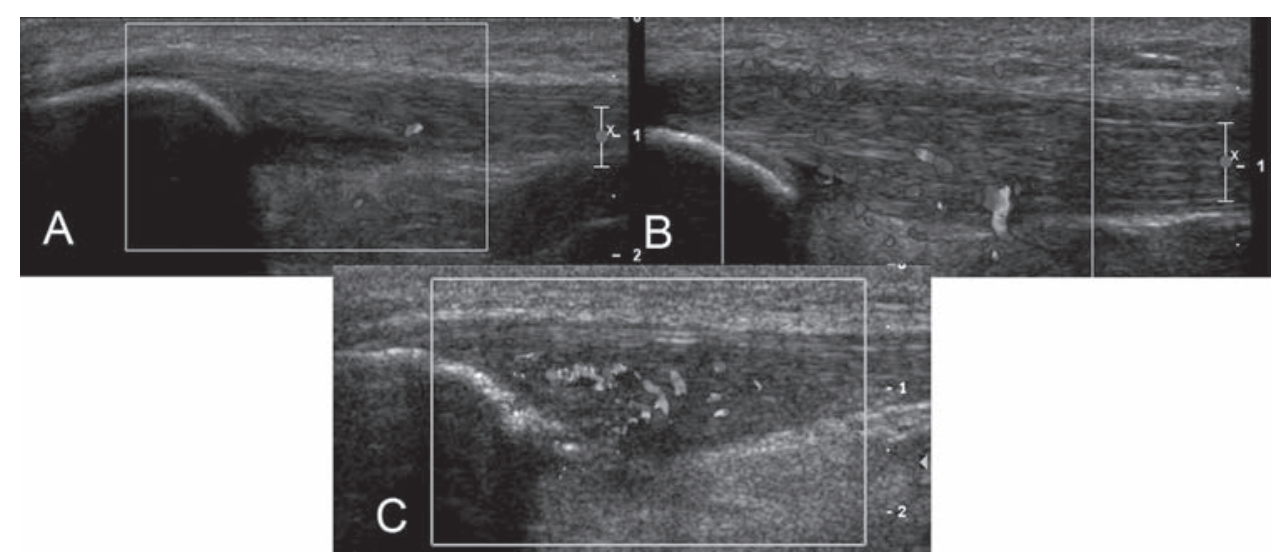

Figure $1(A-C)$ Longitudinal colour Doppler sonogram illustrating the different degrees of neovascularity associated with chronic patellar tendinopathy: (A) grade 1; (B) grade 2 and (C) grade 3. The neovascularity rating used the following scale: '0' represented no blood vessels visible; ' 1 ' indicated mild (one to two vessels extending into the tendon); ' 2 ' moderate (three to four neovessels) and ' 3 ' marked (more than four vessels extending into the tendon). 
in a $2.5 \mathrm{ml}$ syringe) were performed under aseptic conditions using a $27 \mathrm{G}$ needle. Abnormal hypoechoic areas and anechoic clefts/foci in the thickened portion of the patellar tendon were the primary target of the intratendinous injection given under ultrasound guidance using a 7-15 MHz Hockey Stick linear array transducer (Phillips USA, Bothell, Washington, USA). Focal areas of pain were also assessed and targeted. The procedure was considered complete when the participant reported no pain in the infrapatellar tendon. The volume of solution injected varied slightly between tendons depending on the degree of resistance, spread of solution within the tendon and the extent of the abnormality. Approximately $0.5 \mathrm{ml}$ was injected at any one site. Between one and three sites were injected during a given treatment session. The tendon was re-imaged following the injection procedure to assess for spread of the dextrose solution and identify any intrasubstance or partial tears that became more conspicuous following the injection.

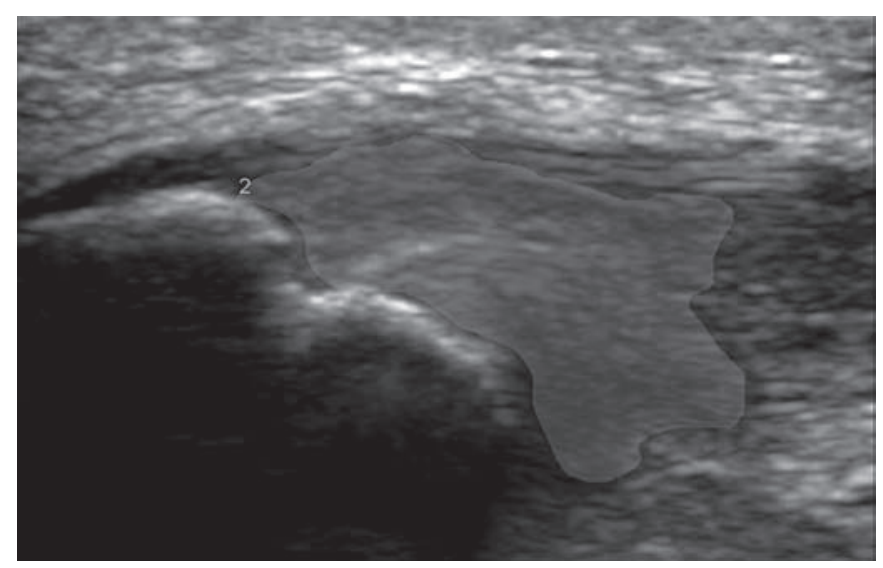

Figure 2 Longitudinal sonogram of the free-hand measurement tool (shaded area) used to measure an area of irregular echo texture (hypoechogenicity).

\section{Follow-up care}

The participant was instructed to refrain from activity that would result in a heavy load to the patellar tendon (ie, jumping or running) during the week following the procedure. Participants were cautioned against taking aspirin or other antiinflammatory agents to relieve any discomfort. Paracetamolbased analgesia was allowed.

The patient was asked to return for follow-up consultation with ultrasound imaging and possible re-injection every 6 weeks until either the patient's symptoms resolved or no improvement was evident, at which time the treatment was discontinued. Patients were encouraged to not seek additional treatments during the follow-up period.

\section{Data collection}

Demographic and baseline status information was collected at the initial consultation. Three visual analogue scale (VAS) pain scores $(100 \mathrm{~mm})$ and ultrasound-based outcomes were recorded at baseline and at the final patient consultation (follow-up). ${ }^{21}$ The VAS items for pain at rest (VAS1), pain during normal daily activity (VAS2) and pain during or after sporting activity (VAS3) were 'do you have infrapatellar pain at rest; do you have infrapatellar pain during activities of daily living (ADL); and do you have infrapatellar pain during or after sporting or other physical activity', respectively.

\section{Data analysis}

Descriptive and mean comparisons of the study data were analysed using SPSS statistical software (copyright 2006, version 15.0.1.). Paired-samples t tests evaluated the change in pain levels from baseline to follow-up. At the follow-up point, VAS pain scores were correlated with documented sonographic features using Pearson's test. Statistical significance for this study was set at a $\mathrm{p}$ value of 0.05 .

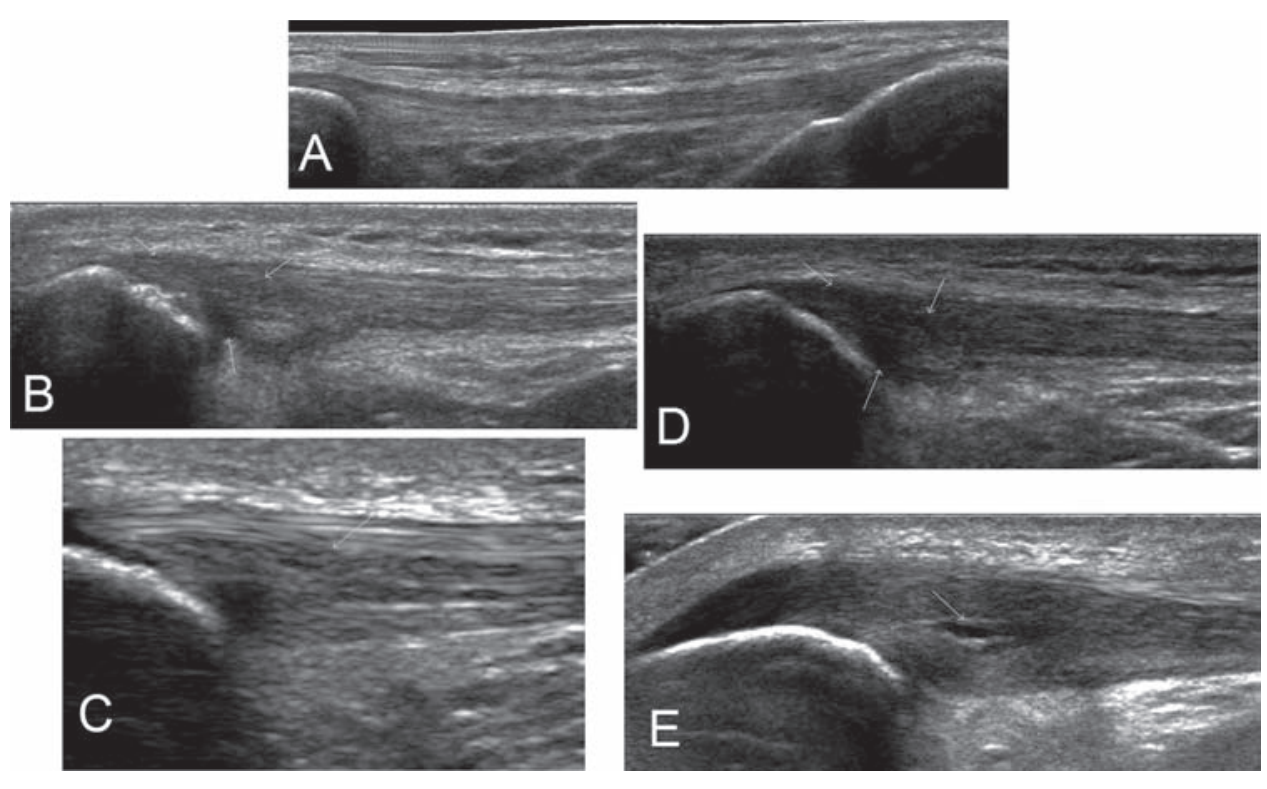

Figure 3 (A-E) Longitudinal sonogram of structural degenerative changes at the patellar tendon during tendinopathy. Slide A shows a normal patellar tendon without marked thickening or hypoechoic changes. Slides B-D illustrate grades 1-3 hypoechogenicity, respectively, with arrow indicating hypoechoic regions. The echotexture rating used a $0-3$ ordinal scale whereby a ' 0 ' represented normal echogenicity and fibrillar pattern, ' 1 ' represented predominately signs of irregular collagen fibre pattern coupled with a slightly weakened echo signal, ' 2 ' represented significant hypoechoic regions and fibre irregularity and ' 3 ' represented marked hypoechogenicity with anechoic regions. Slide E illustrates an intratendinous tear. 


\section{RESULTS}

Forty-seven patellar tendons from 39 male and six female participants (two had bilateral symptoms) aged 38.3 years $( \pm 14.3)$ participated in this prospective case series. The mean reported symptom duration was 21.8 months $( \pm 16.6)$ before enrolment in the study. The median number of injections needed to treat was four ( \pm 3.4 ; range $2-8)$ spaced a mean of 6.4 weeks ( \pm 5.5 days) apart. The final patient consultation, which included follow-up VAS pain and ultrasound examination, took place 45 weeks $( \pm 36)$ post-treatment initiation. None of the participants sought additional treatments during the follow-up period.

VAS pain scores improved significantly $(p<0.01)$ compared with the baseline status (table 1). The VAS score improved by $49 \%$ at rest, $51 \%$ during $\mathrm{ADL}$ and $50 \%$ during sport compared with baseline. Twenty-five participants $(53 \%)$ reported between $50 \%$ and $100 \%$ improvement in pain, 19 cases $(40 \%)$ reported less than $50 \%$ improvement and three cases $(7 \%)$ reported an increase in pain following dextrose injections.

Ultrasound evaluation of the patellar tendon showed structural improvements following dextrose treatment; 12 fewer cases of intratendinous tearing were documented at the 45-week follow-up and eight fewer cases of marked hypoechogenicity (table 2). VAS pain scores at rest, during ADL and

Table 1 A summary of VAS for pain at baseline and at both follow-up points

\begin{tabular}{lll}
\hline & $\begin{array}{l}\text { Baseline VAS }(\mathbf{m m}) \\
(\mathbf{m e a n} \pm \mathbf{S D})\end{array}$ & $\begin{array}{l}\text { 45-Week follow-up } \\
\text { VAS }(\mathbf{m m})(\mathbf{m e a n} \pm \mathbf{S D})\end{array}$ \\
\hline Pain at rest (VAS1)* & $38.4 \pm 25.0$ & $18.7 \pm 18.4$ \\
Pain with daily living (VAS2) $^{*}$ & $51.1 \pm 22.9$ & $25.8 \pm 20.1$ \\
Pain with sport (VAS3)* & $78.1 \pm 15.7$ & $38.8 \pm 26.1$ \\
\hline
\end{tabular}

*Indicates a significant difference in pain score across all time points at $p<0.001$. VAS, visual analogue scale.

Table 2 Overview of continuous, ordinal and dichotomous sonographic variables across time

\begin{tabular}{lcc}
\hline Sonographic feature & Baseline & 45-Week follow-up \\
\hline Tendon thickness $(\mathrm{mm} \pm \mathrm{SD})$ & $8.1 \pm 2.3$ & $8.1 \pm 2.2$ \\
Area of hypoechoic region $\left(\mathrm{mm}^{2} \pm \mathrm{SD}\right)$ & $185.4 \pm 86.5$ & $178.3 \pm 80.4$ \\
Number of tendons with intratendinous & 22 & 10 \\
tear (n) & \multicolumn{2}{c}{} \\
Mean length of intratendinous tear & $6.1 \pm 5.2$ & $6.8 \pm 7.1$ \\
(mm \pm SD) & & \\
Echotexture rating (n) & 0 & 1 \\
Grade 0 & 7 & 17 \\
Grade 1 & 28 & 25 \\
$\quad$ Grade 2 & 13 & 5 \\
$\quad$ Grade 3 & 12 & 19 \\
Neovascularity rating (n) & 13 & 14 \\
$\quad$ Grade 0 & 20 & 13 \\
Grade 1 & 3 & 2 \\
Grade 2 & 21 & 21 \\
$\quad$ Grade 3 & 1 & 1 \\
Intratendinous calcification (n) & & \\
Cortical irregularity (n) &
\end{tabular}

The echotexture rating used a $0-3$ ordinal scale on which ' 0 ' represented normal echogenicity and fibrillar pattern, ' 1 ' represented predominantly signs of an irregular collagen fibre pattern coupled with a slightly weakened echo signal,

' 2 ' represented significant hypoechoic regions and fibre irregularity and

' 3 ' represented marked hypoechogenicity with anechoic regions. The neovascularity rating used the following scale: ' 0 ' represented no blood vessels visible; ' 1 ' indicated mild (one to two vessels extending into tendon); ' 2 ' moderate (three to four neovessels) and ' 3 ' marked (more than four vessels extending into the tendon). during sport also correlated with changes to echotexture severity ( $r$ values $0.306,0.379$ and 0.428 , respectively; $p<0.05$ ); that is, on average, as pain scores decreased, echotexture improved. There were no changes to tendon thickness or hypoechoic areas at follow-up.

Neovascularity also improved. There was a complete elimination of neovessels in seven cases. The greatest reduction came in cases diagnosed at baseline as 'moderate'. There was also no significant association between the degree of neovascularity and VAS scores at follow-up (r values -0.123, -0.011, $0.056 ; \mathrm{p} \geq 0.419$ for rest, $\mathrm{ADL}$ and sport, respectively). There were no changes in any other observed sonographic features. Twenty-one participants showed intratendinous calcification at baseline, which remained unchanged at follow-up.

\section{DISCUSSION}

This prospective pilot level case series reports substantial, consistent improvements in pain outcomes at 45 weeks for dextrose prolotherapy as a treatment for patellar tendinopathy, including an association between VAS score improvement and changes on ultrasound evaluation consistent with tissue healing. The mean reduction in pain scores at rest, with ADL and sport ranged from 20 to $39 \mathrm{~mm}$. Whereas the minimal clinical important difference for patellar tendinopathy using a $100 \mathrm{~mm}$ VAS is not known, this effect size exceeds that of other chronic pain syndromes using a similar outcome measure. ${ }^{22} 23$ These results, in the absence of significant side effects and adverse events, provide a promising indication that dextrose prolotherapy may be effective for patellar tendinopathy. ${ }^{24}$ It is the first study to report the effectiveness of dextrose for patellar tendinopathy and suggests that dextrose prolotherapy improves tendon remodelling on ultrasound imaging.

In addition to improvements in pain in the present study, there were significant changes in the tendon structure and vascularity over the course of treatment. The reduction in both the number of tendons with intratendinous tearing and marked (grade 3 ) hypoechoic regions subsequent to ultrasound-guided dextrose injection treatment suggests tissue remodelling, although histological analysis is needed to confirm this. The significant positive correlation between hypoechoic severity and pain levels at 45 weeks is indirect evidence that degenerative changes such as collagen fibre disruption, fibroblast proliferation or altered morphology, and/or the presence of mucoid ground substance is a source of nociceptive pain in patellar tendinopathy. ${ }^{2}$

Dextrose is hypothesised to initiate a healing response secondary to very local injury tissue perturbation (cell membrane disruption) following a significant change in osmotic pressure between the extracellular matrix and tendon fibroblasts. ${ }^{25}$ Inflammatory cytokines and chemotactic factors are subsequently released from the cell membrane attracting granulocytes and platelets that in turn release pro-healing growth factors. ${ }^{26}$ However, these effects have not been demonstrated compared with control procedures.

Contrary to previous studies, this study did not find an association between neovascularity and pain at the infrapatellar tendon. ${ }^{19} 27$ While there was an overall reduction in the number of cases with moderate to marked neovascularity, seven cases reported being asymptomatic despite also having three or more intratendinous blood vessels observed on ultrasound. Previous research using polidocanol, a sclerosing injectant, has reported a strong association between neovascularity, nerve fibre infiltration and the presence of specific neurotransmitters 
for pain in tendinopathy. ${ }^{28} 29$ The current study is small, however, and the use of colour Doppler (vs power Doppler) may also have compromised our sensitivity in identifying blood vessels within the tendon. ${ }^{30}$ Nevertheless, based on other authors reporting no association between the degree of neovascularity and patient outcomes at the patellar, Achilles and common lateral extensor tendons, it may be reasonable to conclude that neovascularity alone does not reveal an underlying pain mechanism in tendinopathy. ${ }^{31-33}$ Although pain has been associated with neurovascular in-growth, the fact that tendons can be painful or pain-free at any point in their pathogenesis suggests alternative, and currently elusive, causes of pain. ${ }^{16}$

The significant association between hypoechoic severity and pain at follow-up does not indicate causation between the improved echo texture and the reduction in pain; future studies would be needed to support this concept further. It is nevertheless of interest that the findings from the present study are in contrast to Cook et $a^{\beta 4}$ who reported no statistically significant relationship between patellar tendon abnormalities seen on ultrasound and clinical outcomes in elite male athletes. However, differences in the sample population or improvements in ultrasound imaging capabilities may account for these differences.

The degree of pain score improvement in the current study is consistent with that of other therapies for patellar tendinopathy, including eccentric training, ${ }^{35}$ glyceryl trinitrate patches ${ }^{36}$ and injections of either autologous blood ${ }^{37}$ or a sclerosing agent (polidocanol). ${ }^{19}$ Outcomes from these approaches appear promising; reported improvements in pain range from $60 \%$ to $85 \%$, with high patient satisfaction and return to activity reported. For any injection therapy, the effect of the needle itself may be significant, as indicated by outcomes from percutaneous tenotomy procedures for tendinopathy at the infrapatellar, common lateral elbow extensor and Achilles tendon. ${ }^{37-39}$

\section{Limitations and strengths}

Limitations include the lack of a control group and blinding of the radiologist performing the injection/ultrasound assessments. The clinical and ultrasound outcome measures, although relevant to clinical progress and the understanding of the pathophysiology of tendinopathy, have not undergone validation or reliability assessment, nor have they been correlated with histopathological analysis. Documentation of intratendinous tearing may be confounded by the presence of hydrophilic glycosaminoglycans in the extracellular space of the tendon; what therefore may appear as a tear with definitive anechoic boundaries may be degenerative, severely hypoechoic tissue. In either case, the appearance and size of these anechoic regions could be considered a process of disease, and therefore are noteworthy from the standpoint of tissue characterisation following dextrose injection therapy. The follow-up time period after the final injection was relatively short and no specific data on the number of participants who returned to sport are available (although the VAS sport item is meant to reflect real or hypothetical pain experienced during sport and activity). The strengths of the present study include ultrasound-guided needle placement and injection, a large consistent effect size for pain, and improvement in ultrasound outcomes compared with baseline status.

\section{CONCLUSION}

Ultrasound-guided dextrose prolotherapy injections resulted in significant and clinically meaningful reductions in pain in patients with patellar tendinopathy. Tendon structure also improved substantially following injections; changes in hypoechoic severity were significantly correlated with pain outcomes. This suggests that dextrose prolotherapy could be a candidate for non-surgical treatment of refractory patellar tendinopathy. Randomised multidisciplinary effectiveness trials that add validated functional and outcome measures are needed to assess further the utility of dextrose prolotherapy for refractory patellar tendinopathy compared with current therapy.

Funding The authors recognise that financial support for this project was provided by an operating grant from WorkSafeBC.

Competing interests None.

Ethics approval This study was conducted with the approval of the University of British Columbia Clinical Ethics Review Board.

Provenance and peer review Not commissioned; externally peer reviewed.

\section{REFERENCES}

1. Blazina ME, Kerlan RK, Jobe FW, et al. Jumper's knee. Orthop Clin North Am 1973;4:665-78.

2. Khan KM, Bonar F, Desmond PM, et al. Patellar tendinosis (jumper's knee): findings at histopathologic examination, US, and MR imaging. Victorian Institute of Sport Tendon Study Group. Radiology 1996;200:821-7.

3. Alfredson H. The chronic painful Achilles and patellar tendon: research on basic biology and treatment. Scand J Med Sci Sports 2005;15:252-9.

4. Cook JL, Khan KM, Harcourt PR, et al. A cross sectional study of 100 athletes with jumper's knee managed conservatively and surgically. The Victorian Institute of Sport Tendon Study Group. Br J Sports Med 1997;31:332-6.

5. Paavola M, Kannus P, Paakkala T, et al. Long-term prognosis of patients with achilles tendinopathy. An observational 8-year follow-up study. Am J Sports Med 2000;28:634-42.

6. Kettunen JA, Kvist M, Alanen E, et al. Long-term prognosis for jumper's knee in male athletes. A prospective follow-up study. Am J Sports Med 2002;30:689-92.

7. Rees JD, Wilson AM, Wolman RL. Current concepts in the management of tendon disorders. Rheumatology (Oxford) 2006;45:508-21.

8. Dagenais S, Haldeman S, Wooley JR. Intraligamentous injection of sclerosing solutions (prolotherapy) for spinal pain: a critical review of the literature. Spine J 2005;5:310-28.

9. Yelland MJ, Del Mar C, Pirozzo S, et al. Prolotherapy injections for chronic low back pain: a systematic review. Spine 2004;29:2126-33.

10. Topol GA, Reeves KD, Hassanein KM. Efficacy of dextrose prolotherapy in elite male kicking-sport athletes with chronic groin pain. Arch Phys Med Rehabil 2005;86:697-702.

11. Maxwell NJ, Ryan MB, Taunton JE, et al. Sonographically guided intratendinous injection of hyperosmolar dextrose to treat chronic tendinosis of the Achilles tendon: a pilot study. AJR Am J Roentgenol 2007;189:W215-20.

12. Ryan MB, Wong AD, Gillies JH, et al. Sonographically guided intratendinous injections of hyperosmolar dextrose/lidocaine: a pilot study for the treatment of chronic plantar fasciitis. Br J Sports Med 2009;43:303-6.

13. Ryan M, Wong A, Taunton J. Favorable outcomes after sonographically guided intratendinous injection of hyperosmolar dextrose for chronic insertional and midportion achilles tendinosis. Am J Roentgen 2010;194:1047-53.

14. Aström M, Gentz CF, Nilsson P, et al. Imaging in chronic achilles tendinopathy: a comparison of ultrasonography, magnetic resonance imaging and surgical findings in 27 histologically verified cases. Skeletal Radiol 1996;25:615-20.

15. Zeisig E, Ohberg L, Alfredson H. Sclerosing polidocanol injections in chronic painful tennis elbow-promising results in a pilot study. Knee Surg Sports Traumatol Arthrosc 2006;14:1218-24.

16. Cook JL, Purdam CR. Is tendon pathology a continuum? A pathology model to explain the clinical presentation of load-induced tendinopathy. Br J Sports Med 2009;43:409-16.

17. Fornage BD. The hypoechoic normal tendon. A pitfall. J Ultrasound Med 1987:6:19-22.

18. Fornage BD, Rifkin MD. Ultrasound examination of tendons. Radiol Clin North Am 1988;26:87-107.

19. Alfredson $\mathbf{H}$, Ohberg L. Neovascularisation in chronic painful patellar tendinosis - promising results after sclerosing neovessels outside the tendon challenge the need for surgery. Knee Surg Sports Traumatol Arthrosc 2005;13:74-80.

20. Gemignani M, Busoni F, Tonerini M, et al. The patellar tendinopathy in athletes: a sonographic grading correlated to prognosis and therapy. Emerg Radiol 2008;15:399-404. 
21. Boonstra AM, Schiphorst Preuper HR, Reneman MF, et al. Reliability and validity of the visual analogue scale for disability in patients with chronic musculoskeletal pain. Int J Rehabil Res 2008;31:165-9.

22. Kelly AM. The minimum clinically significant difference in visual analogue scale pain score does not differ with severity of pain. Emerg Med J 2001;18:205-7.

23. Farrar JT, Young JP Jr, LaMoreaux L, et al. Clinical importance of changes in chronic pain intensity measured on an 11-point numerical pain rating scale. Pain 2001;94:149-58

24. Ebell MH, Siwek J, Weiss BD, et al. Strength of recommendation taxonomy (SORT): a patient-centered approach to grading evidence in the medical literature. Am Fam Physician 2004;69:548-56.

25. Rabago D, Best TM, Zgierska AE, et al. A systematic review of four injection therapies for lateral epicondylosis: prolotherapy, polidocanol, whole blood and platelet-rich plasma. Br J Sports Med 2009;43:471-81.

26. Anitua E, Andia I, Ardanza B, et al. Autologous platelets as a source of proteins for healing and tissue regeneration. Thromb Haemost 2004;91:4-15.

27. Alfredson $\mathbf{H}$, Ohberg L. Sclerosing injections to areas of neo-vascularisation reduce pain in chronic Achilles tendinopathy: a double-blind randomised controlled trial. Knee Surg Sports Traumatol Arthrosc 2005;13:338-44.

28. Alfredson $\mathbf{H}$, Forsgren S, Thorsen $\mathrm{K}$, et al. In vivo microdialysis and immunohistochemical analyses of tendon tissue demonstrated high amounts of free glutamate and glutamate NMDAR1 receptors, but no signs of inflammation, in jumper's knee. J Orthop Res 2001;19:881-6.

29. Alfredson $\mathbf{H}$, Ohberg L, Forsgren $S$. Is vasculo-neural ingrowth the cause of pain in chronic Achilles tendinosis? An investigation using ultrasonography and colour Doppler, immunohistochemistry, and diagnostic injections. Knee Surg Sports Traumatol Arthrosc 2003:11:334-8.
30. Weskott HP. Amplitude Doppler US: slow blood flow detection tested with a flow phantom. Radiology 1997;202:125-30.

31. Clarke AW, Ahmad M, Curtis M, et al. Lateral elbow tendinopathy: correlation of ultrasound findings with pain and functional disability. Am J Sports Med 2010;38:1209-14.

32. de Vos RJ, Weir A, Cobben LP, et al. The value of power Doppler ultrasonography in Achilles tendinopathy: a prospective study. Am J Sports Med 2007;35:1696-701.

33. Hoksrud A, Ohberg L, Alfredson H, et al. Color Doppler ultrasound findings in patellar tendinopathy (jumper's knee). Am J Sports Med 2008;36:1813-20.

34. Cook JL, Khan KM, Kiss ZS, et al. Asymptomatic hypoechoic regions on patellar tendon ultrasound: a 4-year clinical and ultrasound followup of 46 tendons. Scand J Med Sci Sports 2001;11:321-7.

35. Jonsson P, Alfredson H. Superior results with eccentric compared to concentric quadriceps training in patients with jumper's knee: a prospective randomised study. Br J Sports Med 2005;39:847-50.

36. Murrell GA. Using nitric oxide to treat tendinopathy. Br J Sports Med 2007:41:227-31.

37. James SL, Ali K, Pocock C, et al. Ultrasound guided dry needling and autologous blood injection for patellar tendinosis. Br J Sports Med 2007;41:518-22.

38. McShane JM, Shah VN, Nazarian LN. Sonographically guided percutaneous needle tenotomy for treatment of common extensor tendinosis in the elbow: is a corticosteroid necessary? J Ultrasound Med 2008;27:1137-44.

39. Testa V, Capasso G, Benazzo F, et al. Management of Achilles tendinopathy by ultrasound-guided percutaneous tenotomy. Med Sci Sports Exerc 2002:34:573-80. 


\title{
BJSM
}

\section{Ultrasound-guided injections of hyperosmolar dextrose for overuse patellar tendinopathy: a pilot study}

\author{
Michael Ryan, Anthony Wong, David Rabago, et al.
}

Br J Sports Med 2011 45: 972-977 originally published online February 21, 2011

doi: 10.1136/bjsm.2010.081455

Updated information and services can be found at:

http://bjsm.bmj.com/content/45/12/972.full.html

\begin{tabular}{|c|c|}
\hline \multirow[b]{2}{*}{ References } & These include: \\
\hline & $\begin{array}{l}\text { This article cites } 39 \text { articles, } 20 \text { of which can be accessed free at: } \\
\text { http://bjsm.bmj.com/content/45/12/972.full.htm|\#ref-list-1 }\end{array}$ \\
\hline & $\begin{array}{l}\text { Article cited in: } \\
\text { http://bjsm.bmj.com/content/45/12/972.full.html\#related-urls }\end{array}$ \\
\hline $\begin{array}{l}\text { Email alerting } \\
\text { service }\end{array}$ & $\begin{array}{l}\text { Receive free email alerts when new articles cite this article. Sign up in } \\
\text { the box at the top right corner of the online article. }\end{array}$ \\
\hline
\end{tabular}

Notes

To request permissions go to:

http://group.bmj.com/group/rights-licensing/permissions

To order reprints go to:

http://journals.bmj.com/cgi/reprintform

To subscribe to BMJ go to:

http://group.bmj.com/subscribe/ 